\title{
Medical Image of the Week: Hypertensive Emergencies
}

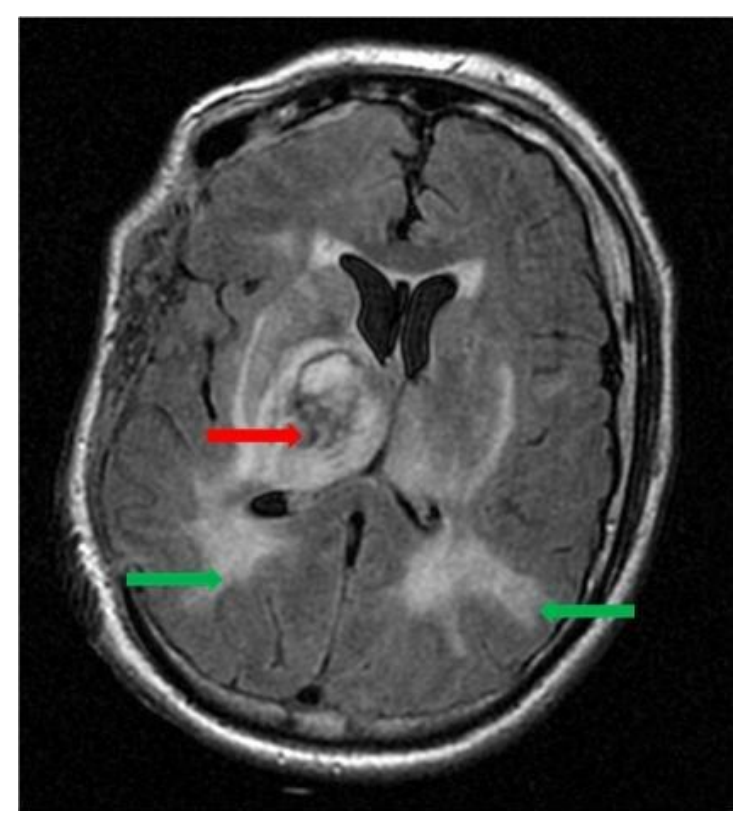

Figure 1. Head CT scan showing basal ganglia hemorrhage (red arrow) and posterior reversible encephalopathy syndrome (green arrows).

A 39-year-old man had sudden onset of left sided hemiparesis, headache and nausea. $\mathrm{He}$ had a history of untreated hypertension and diabetes mellitus. On initial evaluation by emergency medical services, his blood pressure was $270 / 170 \mathrm{~mm} \mathrm{Hg}$. Shortly after admission, he suffered a generalized seizure treated with levetiracetam. His labs were remarkable for a creatinine of $4.4 \mathrm{mg} / \mathrm{dL}$ and microscopic hematuria. His head CT findings are consistent with two simultaneous neurological hypertensive emergencies intracranial hemorrhage of the basal ganglia and posterior reversible encephalopathy syndrome (PRES) (Figure 1) (1). PRES is areas of edema seen as multiple corticosubcortical areas of hyperintense (white) signal involving the occipital and parietal lobes bilaterally and pons. His renal failure likely represents a third hypertensive emergency. His blood pressure was lowered into the 140/90 range within 2 hours by nicardipine infusion and intravenous labetalol boluses. He subsequently suffered worsening mental status and unilateral pupillary dilation and underwent emergent craniotomy. He survived but is currently past 50 days in the hospital.

Robert A. Raschke, MD

Critical Care Medicine

Banner University Medical Center at Phoenix

Phoenix, AZ USA

\section{Reference}

1. Vaughan CJ, Delanty N. Hypertensive emergencies. Lancet. 2000 Jul 29;356(9227):411-7. [CrossRef] [PubMed] 